\title{
Nachdenkliches zur Statistik aus Anlaß eines neuen Gesetzes
}

\author{
Kurt Klein \\ Österreichisches Statistisches Zentralamt, Wien
}

\begin{abstract}
Zusammenfassung: Botschaften aus der Vergangenheit unserer Profession lassen uns manchen Gedanken des neuen Gesetzes als Elemente einer zukünftigen „Statistik-Kultur“ verstehen. Die offenen Fragen ringsum lösen sich dadurch nicht einfach auf.

Der Autor, nach 35 Jahren Tätigkeit im Statistischen Zentralamt (davon 20 Jahre als Abteilungsleiter und die letzten 5 als Vizepräsident) vor dem Ruhestand, sieht dennoch gute Gründe, sich mit diesem Gesetz entschlossen auf den Weg zu machen.
\end{abstract}

\section{Rückblicke}

Knapp vor der Pension neigt man zu Rückblicken. Beginnen wir deshalb mit Geschichte - Zukunft braucht schließlich Vergangenheit als Vorleistung. Die österreichische Statistik hat einige große Zeiten hinter sich. Und die beiden früheren Bundesstatistikgesetze von 1950 und 1965 haben, mindestens partiell, auch zum Aufstieg der Wirtschafts- und Sozialstatistik in den letzten Jahrzehnten beigetragen.

Die erste Phase, an die wir uns erinnern, ist die Frühzeit unserer Profession. Sie reicht von Aufklärung und Josephinismus im späten 18. Jahrhundert bis zu Alphonse Quetelets ,physique sociale“ um die Mitte des 19. Jahrhunderts. Sie reicht von den ersten Statistik-Vorlesungen an den Universitäten bis zur Gründung der ersten statistischen Dienststellen. Statistik war „Aufklärung durch Zahlen“. Es berührt noch heute, mit welcher Leidenschaft, mit welchem Fortschrittsglauben sich unsere beruflichen Vorfahren der frühen Statistik bedient haben, um etwas über Bevölkerungsstrukturen, soziale und wirtschaftliche Verhältnisse, über Arbeiten, Wohnen, Leben und Sterben zu erfahren. „Leidenschaft für die Wirklichkeit“" ist eine Botschaft aus dieser Frühphase unseres Berufs.

Die zweite Phase sind die letzten Jahrzehnte des 19. und der Beginn des 20. Jahrhunderts, als Industrialisierung und soziale Frage in ganz Europa zum Problem wurden. Stichworte wie Verstädterung, Wohnungselend, frühindustrielle Arbeitsbedingungen, der Beginn von Sozialversicherung und Arbeiterschutz können uns an diese Phase erinnern, die von einem Ausbau der Primärstatistik (regelmäßige Volkszählungen, erste Großerhebungen der Wirtschaftsstatistik) begleitet war. Statistik liefert in dieser Phase ein stark erweitertes Spektrum sozialer und wirtschaftlicher Beobachtungen, mit hohen methodischen Standards und einem erstaunlichen Niveau professioneller Reflexion. Die Botschaft an uns: hohe Standards setzen!

Eine dritte Phase waren, trotz unzureichender Mittel, die unruhigen zwanziger Jahre dieses Jahrhunderts, eine kreative Zeit mit neuen Themen und Ansätzen. In dieser bedrängten Zeit wurzeln eine umfassende Lohn-, Preis- und Konjunkturberichterstattung, der Beginn der empirischen Sozialforschung mit Hilfe statistischer Verfahren, graphische 
Methoden zur Visualisierung von Statistik. Kennzeichen dieser Zeit bleibt ein exakter, fast photographischer Zugang der Beobachtung und Beschreibung sozialen Verhaltens (,Die Arbeitslosen von Marienthal“). Die Botschaft an uns: Statistik wird nicht nur „von oben“, sondern auch ,von unten“ gemacht.

Eine vierte Phase haben manche schon bewußt miterlebt: den Wiederaufbau der fünfziger Jahre, verbunden mit einem Neuentwurf der Wirtschaftsstatistik nach dem Regelwerk der Volkswirtschaftlichen Gesamtrechnungen, mit der Grundlage des ersten Bundesstatistikgesetzes von 1950. Schließlich die siebziger Jahre, Blütezeit des „Wohlfahrtsstaates“, einer großzügigen Sozialpolitik, der Sozialforschung und Sozialstatistik - mit der Grundlage des zweiten Bundesstatistikgesetzes von 1965. Mit diesen beiden Entwicklungsschüben waren jene Konturen eines nationalen Statistik-Systems geprägt, mit denen Österreich in die EU gegangen ist.

Die jüngste Phase kennt fast jeder aus eigener Erfahrung: den Eintritt in das straffe supranationale statistische System der EU, das seine Schwerpunkte zunächst bei der Wirtschaftsstatistik zu setzen hatte und nach der ,Re-Sozialisierung“ der siebziger Jahre wieder eine „Re-Ökonomisierung“ der Statistik brachte. Auch diese geänderten Rahmenbedingungen machten ein neues Bundesstatistikgesetz erforderlich.

\section{Einige Folgerungen}

Geschichte ist zum Lernen da. Was können wir aus einem Rückblick lernen? In allen historischen Phasen kommt die Schubkraft der Statistik aus einem deutlichen Wertebezug. Sie begleitet, in einer jeweils ,progressiven“ Position, die großen Entwicklungen durch Beobachtung und Dokumentation von Größenordnungen: Aufklärung und späten Absolutismus, liberalen Verwaltungs- und Beamtenstaat, Kapitalismus und Frühindustrialisierung, bürgerliche und sozialdemokratische Wirtschafts- und Sozialpolitik, die Aufbauphase nach dem Zweiten Weltkrieg, den modernen Leistungs- und Wohlfahrtsstaat, Globalisierung und ,postindustrielle Gesellschaft“. Ökonomische Theoriebildung und VGR gehen eine enge Symbiose ein. Die Humanwissenschaften haben nichts Gleichwertiges anzubieten: Die Bevölkerungs- und Sozialstatistik werden nicht von einer guten Theorie, sondern von der Sogwirkung der Probleme vorangebracht.

Statistik war damit, seit ihrem Beginn, ganz unbefangen ,politische Wissenschaft““. Kritische Köpfe konnten sie jederzeit als „Agentur des Zeitgeistes“ diskreditieren, schlimmer noch: als Legitimation des Bestehenden, als ,konservatives Material“ der politischen Wirklichkeit. Sie ist auch heute als politische Wissenschaft zu verstehen: nicht im Sinne besonderer Politikverbundenheit - wir sind, gerade rund um das neue Bundesstatistikgesetz, von der Politik nicht gerade gestreichelt worden, und Politiker haben ein ambivalentes Verhältnis zu statistischen Daten, besonders wenn sie vorgefaßte Meinungen nicht bestätigen. (Fehlende oder unklare Daten erweitern schließlich politische Handlungsspielräume!)

Aber Statistik ist politisch als Erinnerung an offene Fragen, an aufsteigende Probleme, an Größenordnungen und Proportionen. Und sie ist politisch im Berührtsein von den Dingen und Vorgängen. Statistik wird nicht nur „,von oben“, sie wird auch ,,von unten“ gemacht. Sie vermittelt „Übersicht“ und „Untersicht“. Beim Lesen in alten Publikationen 
merken wir die große Sorgfalt, ein tiefes Ethos, eine fast zärtliche Genauigkeit. Wir spüren den Versuch, mehr Licht über die Dinge zu breiten. Auch das ist gemeint, wenn wir die amtliche Statistik als ,öffentliches Gut“ und damit als Element des politischen Systems einschätzen. „Statistik-Kultur“ gehört zur ,,politischen Kultur“ eines Landes.

Zur ,politischen Kultur“ einer demokratisch verfaßten Gesellschaft gehört die im neuen Gesetz verankerte Unabhängigkeit ihres amtlichen statistischen Dienstes. Es ist kein Zufall, daß nur demokratische Systeme in der Lage sind, hochwertige und unabhängige statistische Informationen zu produzieren, und es ist ebenso kein Zufall, daß viele Statistiken in autoritär geführten Staaten geringere Qualität haben und oft ein selektiv verzerrtes Bild der Wirklichkeit liefern.

Einmal mehr zeigt sich daran: Statistik hat ein demokratisches Grundverständnis“. Sie macht das Unauffällige sichtbar, das Massenhafte, Profane, Gewöhnliche. Statistik ist „Verdichtung des Unscheinbaren“. Eine ,poststatistische Gesellschaft “ paßt verdächtig genau zu manchen anderen ,ppost“-Vorstellungen des gängigen Populismus.

Ein Grundgesetz zur Statistik vermittelt, explizit oder implizit, ein Leitbild von Statistik. Die beiden knappen Gesetze von 1950 und 1965 haben sich mit solchen Ansprüchen nicht abgegeben: Sie sagten, was Statistik tun darf - basta! Freilich - auch dahinter steckt ein Leitbild: Amtliche Statistik ist Informationsdienst für Politik und Verwaltung.

Das neue Bundesstatistikgesetz bringt auch zum Ausdruck, daß die Selbstreflexion der statistischen Dienste und ihre Festschreibung in der Legistik in jüngerer Zeit eine andere Qualität erreicht hat. Amtliche Statistik versteht sich nicht mehr bloß als Datenlieferant für Politik und Verwaltung, sondern als unentbehrliches Element des „Informationsmilieus" demokratisch verfaßter Gesellschaften, als permanente öffentliche Erinnerung an wirtschaftliche und gesellschaftliche Realitäten. Die Gesellschaft braucht Statistik als Information über sich selbst. Deshalb ist Statistik ein zusammenhängendes System von Aufgaben und Lösungen, nicht einfach eine Summe einzelner Erhebungen oder Projekte. Stichworte wie „Grundsätze“, „Qualität“, ,Wissenschaftlichkeit“, ,Professionalität“, Statistik als „öffentliches Gut“, als offenes System für Bürger und Verwaltung sind bei einer Beschreibung amtlicher Statistik heute nicht mehr wegzudenken. Einiges davon hat im neuen Gesetz seinen Niederschlag gefunden.

Wenn ich daran denke, daß die Durchsetzung zentraler fachlicher Anliegen bei der Formulierung des Gesetzes mit politischen und juristischen Standpunkten konkurrierte, muß ich dem Text eine gutes Zeugnis ausstellen. Was, verstreut in einzelnen Bestimmungen, solche Leitgedanken ausdrückt, entspricht dem oben beschriebenen Anspruch und hat internationalen Standard. Dennoch sollte ein Leitbild der Bundesstatistik eigens niedergeschrieben werden. Wenn wir die „Statistik-Kultur“ eines Landes als Teil seiner Standortqualität verstehen, sollte dieses Programm irgendwo nachzulesen sein, in einem Text, der ,ans Hirn und ans Herz“ gehen und deshalb nicht nur aus einer lapidaren Abfolge hierarchisch geordneter Sätze bestehen sollte. Das zuletzt propagierte allgemeine „Leitbild für den Bundesdienst" ist so sehr vom Selbstverständnis der Ministerialbürokratie geprägt, daß sich kaum jemand außerhalb dieser Instanzen darin wiederfinden wird. Auch ein Leitbild der amtlichen Statistik darf nicht zur Selbstreflexion einer Führungsgruppe geraten und sollte für konkrete Ergänzungen ,an der Werkbank“" offengehalten werden.

Wir kommen zum Stichwort „Berufsethik“ - lange verschwiegen, jetzt wieder sehr gefragt, gerade angesichts eines Gesetzes, das trotz hoher Regelungsdichte bestenfalls als 
„Libretto der Zukunft“ gelesen werden kann, zu dem die Musik erst geschrieben werden muß. Dieses Grundgerüst einer ,legalistischen Statistik“ reicht nicht aus: Ich kann meinen Beruf nicht nur als asketischen „Gesetzesvollzug“ realisieren. Berufliche Verantwortung ist eine schöpferische und entdeckerische Aufgabe, ein Appell an mein Leben - ein Leben, das nicht stundenweise verrechnet werden kann. - (Das neue Interesse für Berufsethik entspricht übrigens einer allgemeinen Renaissance von Sinnfragen. Gibt's niemand, der gelegentlich eine „Theologie der Statistik“ schreibt?)

Realitätsbezug ist ein Grundbegriff unseres Berufsethos. Statistik ist ein Instrumentarium für den Umgang mit der Wirklichkeit. Sie vermittelt Einsichten, die sich der Alltagserfahrung entziehen und auch durch andere Zugänge zur Wirklichkeit nicht gewonnen werden können. Arbeit in der Statistik kann deshalb einen besonderen Realitätsbezug vermitteln, der sich durch nüchterne Sachlichkeit, Verständnis für Größen, Proportionen und Zusammenhänge, den Verzicht auf kurzschlüssige Urteile und den Versuch differenzierter Beobachtung und Bewertung auszeichnet.

Andererseits: die amtliche Statistik geht, je nach dem aktuellen Stellenwert von Problemen, mit der realen Welt in ganz unterschiedlicher Dichte, Nähe und Intensität um. Das führt uns zum nächsten zentralen Begriff: Qualität. Der Begriff ist schwer zu fassen, oft nicht meßbar. Viele Konsumenten statistischer Daten verstehen unter Qualität einfach Genauigkeit. Und Scheingenauigkeiten haben bekanntlich etwas Faszinierendes: Die letzte Stelle läßt uns vergessen, daß Statistik eine abstrahierende Wissenschaft ist. Fachstatistiker geraten deshalb oft in die Rolle, nicht für mehr, sondern für weniger, nämlich die jeweils sachgerechte Genauigkeit eintreten zu müssen.

Weitere Kriterien für Qualität: Relevanz, Kohärenz und Konsistenz, Kontinuität, Aktualität, Transparenz, Verfügbarkeit für Bürger und Wissenschaft. Qualitätssicherung ist eine Querschnittsaufgabe. Und sie hängt weitgehend von Metadaten ab. Metadaten sind Informationen über die Daten selbst - Erkenntnis- und „Verdauungshilfen“ für statistische Ergebnisse. Sie müssen ebenso systematisch dokumentiert werden, ebenso zugänglich sein wie die Daten selbst. Gut, daß das neue Gesetz die Publikationspflicht in diesem Sinn versteht. (Eine systematische Dokumentation von Metadaten kann übrigens auch den durch den Computer eingetretenen „Sprachverlust“ von Statistik ausgleichen.) Problem: verschiedene Kriterien für Qualität widersprechen einander (Genauigkeit vs. Relevanz vs. Aktualität). Daher muß es Kompromisse geben. Manchmal fallen sie leicht (z.B. bei der Genauigkeit - absolute Genauigkeit wird selten gebraucht). Manchmal fallen sie schwer, denn Qualität ist kein Lehrbuchthema, sondern ein Kostenfaktor. Positiv, daß das neue Gesetz den Statistikrat als „Qualitätskollegium“vorsieht.

\section{Ausblicke}

Das neue Gesetz gibt der Bundesstatistik einen strikt legalen Rahmen. Es muß dabei den Vollzugscharakter betonen, die Autonomie beschränken. Das ist freilich nur ein Teil der Realität. Statistik muß Probleme antizipieren. Wie soll die Anstalt mit neuen Fragen umgehen, die sich noch nicht zu Gesetzesaufträgen verdichtet haben? Einen Raum für derart „vorgelagertes Handeln“ sieht das Gesetz nicht vor - vielleicht, weil er eine Selbstverständlichkeit ist. Ich bringe einige Themen - vorwiegend aus der Sozialstatistik 
- in Erinnerung, die in diesem ,vorgelagerten“ Rahmen anzugehen sind. Wir nehmen sie als Beispiele, daß sich die Statistik ,visionäre“ Arbeiten durch eine zu enge legalistische Auslegung des Gesetzes nicht verbieten lassen darf.

\section{Sozio-ökonomische Gesamtrechnungen}

In den Bereichen personenbezogener Statistik ist es nicht gelungen, ein den Volkswirtschaftlichen Gesamtrechnungen vergleichbares Rahmenwerk zu schaffen. Dieses Problem wird seit Jahrzehnten bearbeitet, ohne daß die Lösungsansätze befriedigen können. Auf mittlerer Ebene ist freilich durch Sozialdatenhandbücher, Sozialindikatoren, standardisierte Surveys usw. viel vorangebracht worden.

Zusammenfassung und Verdichtung bleiben angesichts der Überproduktion von Einzelstatistiken eine unerläßliche Forderung. Zwei Linien scheinen für die Zukunft wichtig:

1. Direkt an die VGR angeschlossene Satellitenberechnungen in monetären Einheiten (,umweltbereinigtes BIP“, Bewertung von Haushaltsarbeit mit Hilfe von Zeitbudgeterhebungen usw.);

2. Modelle für personenbezogene Gesamtrechnungen in Matrix- oder Kontenform, in denen Daten aus verschiedenen Quellen durch ,statistical matching“ verarbeitet werden (,Social accounting“, ,Labour accounting“).

Von diesen Modellen mit der Einheit „Person“ oder „Arbeitsstunde“ sind auch Verbindungen zu den Geldeinheiten der VGR denkbar. Damit kann die Übergangszone zwischen Bevölkerungs- und Wirtschaftsdaten besser ausgeleuchtet werden.

\section{Sekundärstrukturen}

Viele ,alte“ Themen der Statistik fallen in ihrer Bedeutung zurück oder werden durch neue Entwicklungen abgedrängt: Nebentätigkeiten und Teilzeitarbeit gewinnen an Bedeutung, ohne überall einheitlich erfaßt zu werden. Lebensgemeinschaften können in Volkszählung und Stichproben nur bei gemeinsamem Wohnsitz erfaßt werden. Zweitwohnungen müssen längst in jede Analyse der Wohnversorgung einbezogen werden. Familienarbeit, Soziale Netzwerke und Nachbarschaftshilfe erbringen Leistungen, die schwer erfaßt werden können. Hier gibt es viele Aufgaben für Survey-Erhebungen und Auswertungen aus Verwaltungsregistern.

\section{Marginalisierungsprozesse}

Armut ist in den EU-Staaten wieder zum Thema geworden. Mit der Alterung unserer Bevölkerungen geht eine Zunahme von Behinderungen einher. Prekäre Beschäftigungen (Saison-, Teilzeit-, Wochenendarbeit, Nebentätigkeiten, Werkverträge) ohne volle soziale und arbeitsrechtliche Sicherung breiten sich aus. Hier liegt eine wichtige Aufgabe für Survey-Erhebungen, wobei sich das Europäische Haushaltspanel bereits systematisch des Themas „Armut“ annimmt. 


\section{Verteilungsfragen}

Wenn die Zuwächse geringer werden, nehmen Verteilungskämpfe zu. Auch diese Linie gesellschaftlicher Entwicklung ist von der Statistik zu begleiten. Welche Gruppen stehen „oben“ und „unten“ auf der Wohlstandsskala? Wer sind die Gewinner und Verlierer der letzten Jahre? Lauter Fragen, die sich nach längerem Schweigen wieder zu Wort gemeldet haben und Survey-Erhebungen, Sozialberichterstattung und politische Berichte (z.B. Einkommensbericht zum Bezügebegrenzungsgesetz) deutlich in Anspruch nehmen.

Mikro-Daten zu Verteilungsfragen aus Surveys oder Verwaltungsregistern erfordern überdies die makroökonomische Analyse von Umweltverteilungswirkungen des staatlichen Steuer-, Transfer- und Sozialleistungssystems. Solche Studien enthalten oft überraschende Einsichten: daß der größte Teil gewaltiger Mittelumschichtungen zwischen privaten und öffentlichen Haushalten überhaupt keine oder ganz diffuse Umverteilungswirkungen hat; daß Maßnahmen, die „Umverteilung nach unten“ beabsichtigt haben, im Laufe der Jahre wirkungslos werden können; daß viele Selbstverständlichkeiten in den öffentlichen Budgets, etwa Ausgaben für den Straßenbau, die höheren Schulen und Universitäten, die Kulturförderung oder Wohnbauförderung, auf Seite der Nutzer unvermeidlich „Umverteilung nach oben“ sind. Welche Umverteilungswirkungen Maßnahmen der EU haben, bleibt noch zu untersuchen.

\section{Regionalisierung}

Das Netz europäischer NUTS-Regionen muß, vor allem auf der Ebene NUTS 3, gleichmäßig mit Daten ausgestattet werden - auch im sozialen Bereich. Sind gleichmäßige Lebensbedingungen in verschiedenen Landesteilen ein politisches Ziel, müssen Unterschiede laufend beobachtet und dokumentiert werden. Die Ausbreitung geographischer Informationssysteme läßt die Nachfrage nach kleinräumigen Daten dabei nicht geringer werden. Statistiker sind „,bewußte Europäer“ - der Blick nach Luxemburg und Brüssel bewahrt uns aber nicht davor, in der Regionalstatistik, ,ins Land einizuschaun“.

In der Wirtschaftsstatistik steht die Regionalisierung der Gesamtrechnungen und anderer hochaggregierter Statistiken für die NUTS-Ebenen an erster Stelle. Hier geht es um Förderungen, um Geld für benachteiligte Regionen. Der kleinräumige Nachweis von Arbeitsstätten, Beschäftigung, Pendlerverflechtung, Verkehrsbeziehungen, Emissionen usw. hat gleichfalls hohen Rang. Ein typischer Bereich, der immer wieder kleinräumige Datenmischungen aus Sozial- und Wirtschaftsstatistik verlangt, ist die Marktforschung (Standortuntersuchungen, Arbeitskraftreserven, Verkehrsbedienung usw.).

\section{Emanzipatorische Prozesse}

Sozialstatistik hat seit dem vorigen Jahrhundert emanzipatorische Vorgänge durch Bereitstellung von Daten begleitet. Die Emanzipation der Frauen hat derzeit hohen Stellenwert und erfordert, neben einer Geschlechtsgliederung aller Personentabellen, spezielle Surveys zur weiblichen Familien- und Erwerbsbiographie und systematische Frauen-MännerVergleiche in besonderen Sozialberichten. Daneben ist auf die Lebensverhältnisse von ethnischen Minderheiten, behinderten Personen, Kindern und Jugendlichen usw. aufmerksam zu machen. 


\section{Ausländerpopulationen}

Hohe Ausländeranteile sind zu einem Merkmal vieler europäischer Bevölkerungen und zu einer ,politischen Größe“ geworden. Die gesonderte Darstellung von Ausländerpopulationen wird zu einer Daueraufgabe der amtlichen Statistik. In Demographie und Erwerbsstatistik geschieht dies - Nachholbedarf besteht in den Bereichen Gesundheit, Bildung, Wohnen, Ausstattung und in vielen Bereichen sozialen Verhaltens, die der empirischen Sozialforschung und nicht der Statistik zuzurechnen sind.

\section{Neue Muster sozialer Differenzierung}

Zeitverwendung wird zunehmend zu einem Muster der Charakterisierung sozialer Gruppen - und Zeitbudgeterhebungen werden zu einer Aufgabe amtlicher Statistik. Merkmale der Wohnumgebung werden wichtiger als die ziemlich gleichförmige Innenausstattung der Wohnungen. Die Ausstattung mit aufwendiger Unterhaltungselektronik oder PC liefert gute Unterscheidungsmerkmale, während PKW-Besitz und ähnliches an Unterscheidungswert verloren hat.

\section{Längsschnittbetrachtungen}

De facto gehen wir mit der realen Welt in der Statistik in unterschiedlicher Intensität um. Wir haben ein Nebeneinander von Überfluß und Mangel, von dicht beackerten und EU-geförderten Böden und Brachland, vor allem im Bereich der Bewegungsdaten (flows). Ergänzend zu Querschnitterhebungen sollten deshalb auf der Makro-Ebene „stocks“ und „flows“ modellmäßig in Beziehung gebracht werden, um Strukturen und deren Veränderungen darzustellen. Ähnlich sollten auf der Mikro-Ebene Bildungs-, Erwerbs-, Gesundheits-, Wanderungs- oder Familienbiographien durch Surveys dokumentiert, Berechnungen über Lebenseinkommen oder über die Verteilung der gesamten Lebenszeit angestellt werden. Damit breiten sich Ansätze der Demographie bis in die Wirtschaftsstatistik aus (,Unternehmens-Demographie“). Panel-Erhebungen und RetrospektivSurveys werden zu charakteristischen Instrumenten, Aufstieg und Abstieg (vertikale Mobilität) zu Themen der Statistik.

\section{Dienstleistungssektor}

In fast allen Staaten sind Wirtschaftsstatistik und Konjunkturbeobachtung im Produzierenden Bereich viel dichter als im Dienstleistungssektor. Die Zeiten, in denen die Veränderung der Industrieproduktion als erste Schätzung für die Entwicklung der gesamten Volkswirtschaft herangezogen werden konnte, sind aber vorüber. Ähnliche Indikatoren aus dem Dienstleistungssektor sind rar (Ausnahmen: Handel, Tourismus), obwohl dieser Bereich der Motor für Wachstum und Beschäftigung ist. Relativ einfache Konjunkturerhebungen in den anderen Dienstleistungsbereichen sind unvermeidlich. Es wird nicht ohne Probleme sein, dieses EU-Konzept zu realisieren. Der Produzierende Bereich ist besser auf Statistik ,konditioniert“ als der - meist kleinbetriebliche - Dienstleistungssektor. Damit komme ich gleich zum nächsten Punkt: 


\section{„Statistikfähigkeit“" der Betriebe}

Die Klage über die hohe Belastung der Betriebe durch die laufende Wirtschaftsstatistik ist allgemein, besonders in Ländern wie Österreich, in denen Klein- und Mittelbetriebe dominieren und in denen EU-Qualitätsnormen in vielen Bereichen nur durch Totalerhebungen erreicht werden können. Entgegenkommen müßte hier oft mit Qualitätsverlusten bezahlt werden. Und die Versuchung, unerfüllbare Hoffnungen zu wecken, ist groß. Dennoch scheint, ähnlich wie bei INTRASTAT, die Einführung von (wertschöpfungs-, umsatz-, beschäftigungsabhängigen) Meldeschwellen für laufende Erhebungen vertretbar, wenn periodische Totalerhebungen eine Zuschätzung des fehlenden Teilbereiches ermöglichen. Besonders wichtig: die in Arbeit stehenden ,elektronischen Fragebögen“.

\section{Öffentlicher Sektor}

Der öffentliche Sektor ist ein wichtiger Teil des Dienstleistungsbereiches. In der VGR wird die Wertschöpfung des öffentlichen Sektors durch seine Kosten dargestellt, darunter ein hoher Anteil von Personalkosten. Beschäftigungsreihen für den öffentlichen Dienst haben deshalb auch den Wert von Wirtschaftsindikatoren. Dazu ist es aber erforderlich, Daten aus verschiedenen Quellen zusammenzufassen. Eine gemeinsame Beschäftigtenstatistik der „öffentlichen Hände“ fehlt, die Makro-Operationen im Rahmen der VGR können auf Strukturdaten wenig Rücksicht nehmen. Besser gegliederte und besser vergleichbare Beschäftigungsdaten des öffentlichen Sektors sind unentbehrlich.

\section{Grund und Boden}

Von den drei „klassischen“ Produktionsfaktoren Grund und Boden, Arbeit und Kapital wissen wir über Arbeit am meisten, über die beiden anderen zu wenig. Für Finanzierungsund Geldstromrechnungen sind die Zentralbanken der bessere Adressat. Über Grund und Boden können wir nicht schweigen. Dazu haben wir, kein Zweifel, eine Reihe von Informationen: aus dem Kataster über Flächen, Nutzungsformen und Eigentümer, aus der Agrarstatistik über Anbauflächen und Erträge, aus der Flächenwidmung über Baulandreserven, aus dem Umweltbereich über Bodenqualität und Immissionen usw. Was es nicht gibt, ist ein Datenverbund, der diese Informationen zusammenbringt. Ein solches System ist seit Jahrzehnten eine Forderung von Experten und bleibt auch an der Jahrtausendwende ein Wunschtraum. Trotzdem müssen wir festhalten: Boden ist ein knappes Gut, und wenn wir sorgsam und geordnet damit umgehen wollen, brauchen wir bessere Informationen.

\section{Schluß}

Das neue Bundesstatistikgesetz ist viel länger und komplizierter als seine Vorgänger. Nehmen wir's als Symbol: Statistik ist schwieriger geworden. Die Entdeckerfreude der frühen Jahre, viel unbefangene Sicherheit ist verlorengegangen. Statistik kann Sicherheit vermitteln: mit jeder Tabelle kann ein Problem durchsichtiger, ein Stück Wirklichkeit überschaubarer werden. Aber je dichter die Welt mit Statistik besetzt ist, desto widersprüchlicher wird Statistik selbst - ihre Funktion im System, manches an ihren Begriffen 
und Ergebnissen. Deshalb reizt Statistik zum Widerspruch: „I have made up my mind don't confuse me with facts!“ Die eigene, affektiv und ideologisch verzerrte Erfahrung wird trotzig gegen das intersubjektive Wissen gestellt, das Statistik vermittelt. Statistik hat deshalb unverdrossen ihr Zeugnis für die Wirklichkeit abzulegen - auch gegen uns selbst, unsere Vorurteile und unvollständigen Erfahrungen.

Das neue Gesetz hat die Situation rund um das auslaufende Zentralamt zunächst einmal beruhigt. Die Aufregungen vor etwa drei Jahren sind nicht vergessen, aber an den Rand getreten. Abwarten dominiert, manchmal leise Hoffnung. Dabei müssen wir sagen: gut, daß Statistik noch jemand aufregt! Größer ist die Gefahr, daß wir immer mehr produzieren und daß das niemand mehr aufregt. Statistik soll „,ie Zahlen zum Reden bringen“ - der Umgang mit der Sprache zählt nicht immer zu den Stärken unserer Profession. Ich bin nicht ohne Sorge, daß uns damit auch ein Stück Identität verlorengeht - unsere Erinnerung, wo wir herkommen und hingehören. Statistik besteht nicht nur aus Maschinen, Methoden und Mathematik. Wir brauchen Menschen, die nicht nur Termine, sondern die vor allem Wirklichkeit wahrnehmen und interpretieren können.

Angesichts des neuen Gesetzes haben viele den Eindruck: das große Zeichen über unserer Zukunft ist das Fragezeichen. Ich konnte zur Sache auch nur einige angefangene Gedanken beisteuern. Und ich gehöre nicht zu jenen Leuten, die immer ganz genau wissen, wofür die Zeit reif ist und was daher zu geschehen hat. Aber ich meine, daß wir uns nicht verabschieden sollten, ohne einander Mut zu machen. Statistik hat Zukunft! Auch bei knappen Ressourcen müssen wir aus der Defensive herausfinden. Prioritäten setzen, nicht auf Expansion, sondern auf Qualifizierung vertrauen! Systemorientiert arbeiten keine „Partikelverehrung“! Lassen wir uns vom Alltag nicht völlig verbrauchen - Statistik bleibt eine farbige, vitale Sache! Ein Gesetz, das bisher nur auf dem Papier des Bundesgesetzblattes existiert, soll mit uns und durch uns ein Stück Wirklichkeit werden. Ich habe dazu große Zuversicht, weil ich genügend Leute kenne, denen bei der täglichen Arbeit die Leidenschaft für ihre Aufgabe nicht abhanden gekommen ist.

Adresse des Autors:

Vizepräsident Hofrat Dr. Kurt Klein

Österreichisches Statistisches Zentralamt

Hintere Zollamtstraße 2b

A-1033 Wien, Österreich

Tel.: +431711287891

Fax: + 4317156828

Email: KKlein@oestat.gv.at 
